ORIGINAL ARTICLE

\title{
Association between Sucrose Intake in Coke and Root or Coronal Caries among Teenagers
}

\author{
SYED AMMAR HAIDER ${ }^{1}$, MOHAMMAD IRFAN ${ }^{2}$, AMINA RASHID ${ }^{3}$, ANOOSHAY EJAZ ${ }^{4}$, AFRA ARSLAN ${ }^{5}$, MUHAMMAD \\ HASSAN $^{6}$, AMINA TARIQ ${ }^{7}$ \\ ${ }^{1}$ Islamic International Dental College Islamabad, Riphah International University \\ ${ }^{2}$ Islamic International Dental College, Riphah International University \\ ${ }^{3}$ Demonstrator, Department of Community and Preventive Dentistry, Akhter Saeed Medical \& Dental College, Lahore. \\ ${ }^{4}$ Postgraduate Resident, Department of science of dental materials, de Montmorency College of Dentistry, Lahore \\ ${ }^{5}$ General Dentist, University College of Medicine \& Dentistry, University of Lahore \\ ${ }^{6}$ Associate professor, Department of Science of Dental Materials, University College of Medicine \& Dentistry, University of Lahore \\ ${ }^{7}$ Postgraduate Research Coordinator, University College of Medicine \& Dentistry, University of Lahore, \\ Correspondence to Amna Tariq, Email id: aminatariq8@gmail.com
}

\begin{abstract}
Aim: To examine the associations between sucrose intake in coke and the prevalence of root or coronal caries in teenagers.

Methods: Crossectional survey design was used to achieve the aim of the study. Participants with the age ranging from 13 to 19 years were recruited using consecutive sampling technique. 60 participants were recruited in the study who take coke as carbonated drink regularly.

Results: The results revealed that there was a significant difference in the prevalence of teeth with root caries or restoration in terms of levels of intake of sucrose in coke $\left(X^{2}=6.205, P=.045\right)$. Teeth with root caries or restoration were found higher among participants with medium sucrose intake in the form of coke (58.33\%). Teeth with coronal caries or restoration were found higher among participants with high sucrose intake in the form of coke (66.66\%).

Conclusion: A significant positive relationship was observed between sucrose intake in coke and the number of coronal or root caries lesions in teenagers

Keywords: Root caries, Coronal caries, Sucrose intake, Coke
\end{abstract}

\section{INTRODUCTION}

The most prevalent dental problem which is noncommunicable is caries around the globe. Dental caries is a significant problem dealt in public health till present. According to the findings reported, loss of tooth is also linked with the carious tooth ${ }^{1}$. So, it is important to explore the causes of dental caries especially in low income counties where first aid is also not commonly available.

It is significantly required to explore the linkage between the food habits, nutritional levels and occurrence of dental caries among teenagers. As per earlier literature available, intake of fruit, vitamins, dietary fibre, vegetable, and minerals are associated with dentation. It was found that individuals wearing dentures face difficulties in eating and thus been able to consume important nutrients as they have less food choices ${ }^{2,3}$. Dairy products such as milk and products made of milk are found to be linked to plays a preventive role in development of dental caries as higher level of calcium and protein is present in milk ${ }^{4}$. According to Gueguen and Pointillart ${ }^{5}$, other reason for playing preventive role in dental caries is that most of the times milk is available as fortified with vitamins $A$ and $D$ which is helpful and beneficial for absorption of calcium at higher level.

It is a fact that avoiding glucose, fructose etc. could be helpful in prevention of dental caries. Currently, it was reported in the guidelines of the World Health Organization (WHO) mentioning that the free sugar intake should provide intake of energy upto $\leq 10 \%$ and recommended

Received on 17-01-2021

Accepted on 25-05-2021 additional declines upto $<5 \%$ to prevent dental health during the course of life. These recommendations were reported by Moynihan in 2016 after conducting a systematic review based on the evidence concerning the intake of sugar in association to risk of dental caries prevalence. Intake of starch and its association with the risk of occurrence of dental caries was not found as a result of conducting a systemic review but it was recommended that higher intake of starch which has ability to quickly digestible may elevate the caries risk. ${ }^{6}$ Sweet choices as well as consumption of sweet in the form of coffee and tea are associated with the experience of dental caries as reported in many studies $7,8,9,10,11,12$.

A study conducted on intake of carbonated drinks and dental erosion reported that there is a strong association of carbonated drinks intake and dental erosion in university students in Pakistan ${ }^{13}$.

According to our knowledge and literature reviewed there are only little research has done on impact of sucrose intake in Coke on dental caries prevalence, particularly root caries among teenagers in Lahore Pakistan. Thus, this study aimed to explore the correlation between the sucrose intake, and the occurrence of root or coronal caries in among teenagers in Lahore Pakistan.

\section{METHODOLOGY}

Cross-sectional survey design was used to achieve the aim of the study. Participants with the age ranging from 13 to 19 years were recruited using consecutive sampling technique. 60 participants were recruited in the study who take coke as carbonated drink regularly. 
Survey tools: Questionnaire contained brushing habits (1 times a day, 2 times a day, not daily), and dental checkups (yes/ no).

Oral examination: Team of trained dentists examined the status of oral health. Using artificial light, with mirror and ball-point periodontal probes, dental examination was done to explore the prevalence of caries. Using criteria recommended by WHO in $1997^{14}$, the root caries were diagnosed. At first, it was determined that root surface must be visible at least up to $1 \mathrm{~mm}$ between restoration margin and crest of gingiva. Decay of root was known as a lesion present on the exposed surface of root which was found soft on probing.

Where tooth decay affected the root as well as crown, the expected site of lesion origin was documented as decayed. In case of not been able to judge the site of lesion origin, the crown as well as the tooth root were documented as decomposed. In fillings cases where both the root and crown were involved were taken as the highly expected primary carious lesion site as filled. At the time when judgment about origin site was not possible, it was documented that the crown as well as the root was filled. A multiple-surface lesion or filling was recorded if it extended more than one-third of the way across the adjacent surface. Coronal decay was defined as an unmistakable cavity, undermined enamel, or a detectable soft lesion.

The researchers were rectified with 60 volunteer patients at the University Hospital before the study. Assessment of inter-examiner reliability for surfaces (root caries) and teeth (coronal caries) was performed. Kappa values among each pair of examiners were $0.70-0.83$ for coronal caries and for exposed root caries it was $0.77-$ 0.86 .
Statistical Analysis: Data were entered into SPSS version 23.0 for data analysis. Participants were categorized in terms of intake of sucrose in coke as low, medium and high intake. One Way ANOVA was used to compare the mean values between the three groups. Chi-Square test was used to explore the difference between the intake of sucrose in coke and occurrence of root or coronal caries.

\section{RESULTS}

The results of chi-square revealed that majority of males reported medium intake of sucrose $19(52.8 \%)$ whereas majority of females have high sucrose intake in coke $12(50 \%)$. But in comparison, females take more sucrose in the form of coke as compared to males $\left(X^{2}=9.016, P=.011\right)$. Smokers who take higher amount sucrose in the form of coke were only $6(20 \%)$ of the whole data $\left(X^{2}=26.0\right.$, $\mathrm{P}<.001)$. Participants with medium intake of sucrose are found more in having habits of cleaning and scaling of teeth $18(50 \%)$ as compared to participants with higher and lower intake of sucrose in coke $\left(X^{2}=7.917, P=.019\right)$. The usage of interdental aids was found higher among participants whose intake of sucrose in coke was at medium level as compared to low and high intake $\left(\mathrm{X}^{2}=26.0, \mathrm{P}<.001\right)$.

The results revealed that there was a significant difference in the prevalence of teeth with root caries or restoration in terms of levels of intake of sucrose in coke $\left(X^{2}=6.205\right.$, $P=.045)$. Teeth with root caries or restoration were found higher among participants with medium sucrose intake in the form of coke $(58.33 \%)$. Teeth with coronal caries or restoration were found higher among participants with high sucrose intake in the form of coke $(66.66 \%)$

Table 1: Comparison of Demographics with Sucrose Intake

\begin{tabular}{|c|c|c|c|}
\hline \multirow{2}{*}{ Gender } & \multicolumn{3}{|l|}{ Sucrose Intake } \\
\hline & Low sucrose intake & Medium sucrose intake & High sucrose intake \\
\hline Male & $11(30.6 \%)$ & $19(52.8 \%)$ & $6(16.7 \%)$ \\
\hline Female & $7(29.2 \%)$ & $5(20.8 \%)$ & $12(50.0 \%)$ \\
\hline \multicolumn{4}{|c|}{$X^{2}=9.016, P=.011$} \\
\hline \multicolumn{4}{|c|}{ Smoking } \\
\hline No & $0(0 \%)$ & $18(60 \%)$ & $12(40 \%)$ \\
\hline Yes & $18(60 \%)$ & $6(20 \%)$ & $6(20 \%)$ \\
\hline \multicolumn{4}{|c|}{$\mathrm{X}^{2}=26.0, \mathrm{P}<.001$} \\
\hline \multicolumn{4}{|c|}{ Cleaning and scaling } \\
\hline Yes & $6(16.7 \%)$ & $18(50 \%)$ & $12(33 \%)$ \\
\hline No & $12(50 \%)$ & $6(25 \%)$ & $6(25 \%)$ \\
\hline \multicolumn{4}{|c|}{$\mathrm{X}^{2}=7.917, \mathrm{P}=.019$} \\
\hline \multicolumn{4}{|c|}{ Inter Dental Aid } \\
\hline No & $18(60 \%)$ & $6(20 \%)$ & $6(20 \%)$ \\
\hline Yes & $0(0 \%)$ & $18(60 \%)$ & $12(40 \%)$ \\
\hline
\end{tabular}

Table 2: comparison of sucrose intake with teeth with root caries, coronal caries or restoration

\begin{tabular}{|l|l|l|l|}
\hline $\begin{array}{l}\text { Teeth with root caries or } \\
\text { restoration }\end{array}$ & Sucrose Intake & Medium sucrose intake & High sucrose intake \\
\cline { 2 - 4 } & Low sucrose intake & $14 / 24$ & $10 / 18$ \\
\hline Yes & $4 / 18$ & $10 / 24$ & $8 / 18$ \\
\hline No & $14 / 18$ & \multicolumn{2}{|l|}{} \\
\hline $\mathrm{X}^{2}=6.205, \mathrm{P}=.045$ & \multicolumn{3}{|l|}{} \\
\hline Teeth with coronal caries or restoration & $12 / 24$ & $6 / 16$ \\
\hline No & $0 / 18$ & $12 / 24$ & $12 / 18$ \\
\hline Yes & $18 / 18$ & & \multicolumn{2}{|l|}{} \\
\hline $\mathrm{X}^{2}=12.381, \mathrm{P}=.002$ &
\end{tabular}




\section{DISCUSSION}

The findings of this study on the teenagers in Pakistan, higher sucrose in the form of coke was correlated with higher occurrence of root and coronal caries as mentioned in table 2. The results suggest that higher sucrose intake in the form of coke might raise the possibility of occurrence of root or coronal caries. The current research has shown that sucrose in coke was highly related with caries in teeth. Teenagers must focus on this matter to reduce usage of coke to prevent from caries as it was reported that sucrose intake at any level is potentially cariogenic in nature. ${ }^{15}$ The relationship between total sugar intake and dental caries was reported by TSO, in $2015^{16}$.

While reporting the guidelines of WHO (2015) on free sugars, in a systematic review it was reported that sugar consumption frequency is highly associated with risk of dental caries occurrence ${ }^{17,18}$. A systematic review of numerous existing human epidemiologic research explored that the sucrose intake frequency is a significant fundamental issue for development of dental caries. ${ }^{18}$ Nine or more than 9 sucrose intakes was reported by Steele et al. ${ }^{19}$ which increase the risk of developing dental caries by two folds. The guidelines provided by WHO (2015) reported that by limiting the intake of sucrose can reduce the risk of dental caries prevalence ${ }^{17}$.

On contrary to the current findings, a study reported that there was no association of sucrose intake with root caries (Christensen et al., 2015) ${ }^{20}$. Even though relationship was not reported but researcher could not eradicate the likelihood of a result reporting bias.

The limitation of this study was only sucrose in coke was focused while not controlling the intake of other sugary foods.

\section{CONCLUSION}

The study findings revealed a positive relationship between the sucrose intake in the form of coke and root or coronal caries in teenagers in Pakistan. Further future studies as well as clinical trials will be of great important in terms of need of hour to assess the association of sucrose and root or coronal caries occurrence.

\section{REFERENCES}

1. Gregory, D. and Hyde, S. (2015): Root Caries in Older Adults. Journal of the California Dental Association 43, 439-445.

2. Su Y, Yuki M, Hirayama K, Sato M, Han T. Denture Wearing and Malnutrition Risk Among Community-Dwelling Older Adults. Nutrients. 2020 Jan 5;12(1):151. doi: 10.3390/nu12010151. PMID: 31948104; PMCID: PMC7020032.

3. Grammatikopoulou MG, Gkiouras K, Theodoridis X, Tsisimiri M, Markaki AG, Chourdakis M, Goulis DG. Food insecurity increases the risk of malnutrition among community-dwelling older adults. Maturitas. 2019 Jan;119:8-13. doi: 10.1016/j.maturitas.2018.10.009. Epub 2018 Oct 28. PMID: 30502753.

4. Heaney, R.P. (2000): Calcium, dairy products and osteoporosis. Journal of the American College of Nutrition 19, 83s-99s.

5. Gueguen, L. and Pointillart, A. (2000): The bioavailability of dietary calcium. Journal of the American College of Nutrition $19,119 \mathrm{~s}-136 \mathrm{~s}$

6. Halvorsrud, K., Lewney, J., Craig, D. and Moynihan, P.J. (2018): Effects of Starch on Oral Health: Systematic Review to Inform WHO Guideline. Journal of Dental Research 98(1), 46-53.

7. Gudkina, J. and Brinkmane, A. (2010): The impact of salivary mutans streptococci and sugar consumption on caries experience in 6-year olds and 12-year olds in Riga. Stomatologija 12, 56-59.

8. Gudkina, J., Brinkmane, A., Abrams, S.H. and Amaechi, B.T. (2016): Factors influencing the caries experience of 6 and 12 year old children in Riga, Latvia. Stomatologija 18, 14-20.

9. Jamel, H.A., Sheiham, A., Watt, R.G. and Cowell, C.R. (1997): Sweet preference, consumption of sweet tea and dental caries; studies in urban and rural Iraqi populations. International Dental Journal 47, 213-217.

10. Karki, S., Pakkila, J., Ryhanen, T., Laitala, M.L., Humagain, M., Ojaniemi, M. and Anttonen, V. (2019): Body mass index and dental caries experience in Nepalese schoolchildren. Community Dentistry and Oral Epidemiology 47, 346-357.

11. Maru, A.M. and Narendran, S. (2012): Epidemiology of dental caries among adults in a rural area in India. The Journal of Contemporary Dental Practice 13, 382-388.

12. Montero, J., Costa, J., Bica, I. and Barrios, R. (2018): Caries and quality of life in portuguese adolescents: Impact of diet and behavioural risk factors. Journal of Clinical and Experimental Dentistry 10, e218-e223.

13. Khan S, Khalid N, Maqsood S, Tariq A, Hassan M. Association of Carbonated Drinks intake with Dental Erosion among Dental Students: A Comparison between Day Scholars and Hostel Residents. International Journal of Contemporary Medical Research [IJCMR]. 2020;7(4).

14. WHO (1997): Oral Health Surveys - Basic Methods, 4th ed. Geneva: World Health Organization.

15. Mela, D.J. and Woolner, E.M. (2018): Perspective: Total, Added, or Free? What Kind of Sugars Should We Be Talking About? Advances in Nutrition 9, 63-69.

16. The Stationery Office (2015): Scientific Advisory Committee on Nutrition Carbohydrates and Health. London: TSO.

17. WHO (2015): WHO Guideline: sugars intake for adults children. Geneva: WHO.

18. Moynihan, P.J. and Kelly, S.A. (2014): Effect on caries of restricting sugars intake: systematic review to inform WHO guidelines. Journal of Dental Research 93, 8-18.

19. Steele, J.G., Sheiham, A., Marcenes, W., Fay, N. and Walls, A.W. (2001): Clinical and behavioural risk indicators for root caries in older people. Gerodontology 18, 95-101.

20. Christensen, L.B., Bardow, A., Ekstrand, K., Fiehn, N.E., Heitmann, B.L., Qvist, V. and Twetman, S. (2015): Root caries, root surface restorations and lifestyle factors in adult Danes. Acta Odontologica Scandinavica 73, 467-473. 